\title{
Electrical Potential Difference Across the Nasal Epithelium Is Reduced in Premature Infants With Chronic Lung Disease but Is Not Associated With Lower Airway Inflammation
}

\author{
EROL A. GAILLARD, NIGEL J. SHAW, HELEN L. WALLACE, GILL VINCE, AND KEVIN W. SOUTHERN
}

Institute of Child Health [E.A.G., H.L.W., K.W.S.], University of Liverpool, Royal Liverpool Children's Hospital, Liverpool L12 2AP, United Kingdom; Neonatal Unit [N.J.S.], Liverpool Women's Hospital, Liverpool L8 7SS, United Kingdom; Department of Immunology [G.V.], University of Liverpool, L69 3GA, United Kingdom

\begin{abstract}
Airway liquid content and insufficient absorptive airway ion transport at birth are potentially important factors in the development and severity of neonatal respiratory disease. The role of deficient absorptive airway ion transport in the development of chronic lung disease of prematurity is unknown. Additionally, lung inflammatory mediators modulate airway ion transport. Their effect on preterm lung ion transport and absorptive capacity is not established. We performed serial nasal potential difference studies and broncho-alveolar lavage in preterm infants born less than $30 \mathrm{wk}$ postmenstrual age over the first four postnatal weeks. Our study aims were to: 1) compare nasal potential difference between preterm infants developing chronic lung disease and babies of similar gestation who do not; and 2) examine for an association between airway inflammation and ion transport parameters. We found that potential difference across the nasal epithelium increased with gestation, remained low and unchanged in infants developing chronic lung disease over the first four postnatal weeks, was significantly lower at four weeks in chronic lung disease infants, and was not associated with lower airway inflammation at any time point. We conclude that infants with chronic lung disease postnatally have a persistently reduced absorptive airway ion transport capacity. (Pediatr Res 61: 77-82, 2007)
\end{abstract}

$\mathrm{T}$ he incidence of chronic lung disease of prematurity (CLD) in very preterm infants has not changed significantly over the last decade despite the routine administration of antenatal steroids and postnatal exogenous surfac$\operatorname{tant}(1,2)$.

A greater residual airway liquid content resulting from incompletely developed absorptive airway ion transport processes at birth is a potentially important factor in the pathogenesis and severity of the neonatal respiratory distress syndrome (RDS) (3-5). Compared with preterm babies without neonatal lung disease, those with RDS have an increased lung liquid content $(6,7)$.

\footnotetext{
Received April 4, 2006; accepted September 5, 2006

Correspondence: Erol A. Gaillard, Ph.D., Respiratory Department, Royal Liverpool Children's Hospital, Eaton Road, GB-Liverpool, L12 2AP; e-mail: gaillard@liv.ac.uk

Dr Gaillard was supported by the Liverpool Women's Hospital Newborn Appeal. Dr Wallace was supported by the Medical Research Council, UK.
}

DOI: $10.1203 / 01 . p d r .0000250035 .10339 . c e$
Lung inflammation is present in preterm infants with RDS $(8,9)$. Those who develop CLD experience a persistent and greater lung inflammatory response $(10,11)$.

Recent studies have demonstrated that inflammatory mediators influence airway ion transport processes (12-16). However, in vitro studies have given conflicting results with respect to the effect of cytokines on airway ion transport processes. Interleukin (IL)- 4 and interferon- $\gamma$ inhibit the amiloride sensitive epithelial sodium channel $(\mathrm{ENaC})(12,13)$ in polarized cultured human bronchial epithelial cells whereas tumor necrosis factor (TNF)- $\alpha$ was not found to modulate ion transport (13). In vivo experiments have demonstrated a TNF- $\alpha$ (14), IL-1 $\beta$ (15) and TGF- $\alpha$ (16) mediated increase in lung liquid absorption. These studies were conducted with single cytokines in models of mature lung epithelium. There are no data on the impact of cytokines on the airway ion transport processes of the human newborn lung in vivo.

The aim of this study was to examine in vivo the association between cytokine concentration in lung lavage fluid and absorptive airway ion transport in human preterm babies.

We used serial matched nasal potential difference (PD) measurements with concomitant lung fluid cytokine estimation during the first four postnatal weeks in babies born below 30 wk postmenstrual age (wpma). Our hypotheses were that absorptive airway ion transport capacity increases with gestational age, is lower in infants with CLD and is reduced in association with airway inflammation.

\section{METHODS}

The local research ethics committee approved the study. Infants were recruited between January and December 2002. Informed written consent was obtained from the parents.

Subjects. Babies born below 30 wpma at Liverpool Women's Hospital or transferred postnatally (within $24 \mathrm{~h}$ of birth) to the Neonatal Unit were eligible for enrolment into the study. Babies with chromosomal abnormalities, congenital cardiac disorder other than persistent ductus arteriosus (PDA), diaphragmatic hernia and infants from families with a child known to have cystic fibrosis were excluded.

A diagnosis of RDS was based on clinical presentation and review of the chest radiograph. Infants requiring ventilatory support or supplemental oxy-

Abbreviations: PD, potential difference; ENaC, epithelial sodium channel; RDS, respiratory distress syndrome; CLD, chronic lung disease of prematurity; BAL, broncho-alveolar lavage; IQR, interquartile range; Wpma, weeks postmenstrual age 
gen at 36 wk corrected postmenstrual age were deemed to have CLD. Oral intubation and mechanical ventilation was the preferred mode of ventilatory support at Liverpool Women's Hospital during the study period. Continuous positive airway pressure ventilation was used infrequently. Supplemental oxygen for self-ventilating patients was delivered if peripheral oxygen saturations were $<89-94 \%$ in babies $<34$ wk corrected gestation and $\leq 94 \%$ in babies $\geq 34$ wk corrected gestation.

Nasal PD measurement. Nasal PD was measured within $24 \mathrm{~h}$ of birth, at median postnatal day 3,8 and 29. The nasal PD measurement has been described in detail elsewhere $(17,18)$. Briefly, the PD was measured between an exploring electrode and an intravenous reference electrode moved along the lateral floor of the nose.

Three outcome measures were obtained. A stable maximal baseline PD was recorded during perfusion of the nasal epithelium with physiologic saline solution $\left(147 \mathrm{mM}\right.$ of $\mathrm{Na}^{+}$and $156 \mathrm{mM}$ of $\left.\mathrm{Cl}^{-}\right)$at a rate of $0.1 \mathrm{~mL} / \mathrm{min}$. From this point two further measurements were taken.

The change in PD following perfusion with a physiologic saline solution containing amiloride $10^{-4} \mathrm{M}(\delta$-amil).

The change in PD following perfusion with a solution in which $\mathrm{Cl}^{-}$was replaced by gluconate and amiloride $10^{-4} \mathrm{M}$, which allows to measure the capacity of the proximal airway epithelium to secrete $\mathrm{Cl}^{-}\left(\delta\right.$ zeroCl$\left.{ }^{-}\right)$.

Measurements were performed in one nostril. Subsequent measurements were then taken from the same nostril whenever possible.

Broncho-alveolar lavage. BAL fluid was obtained daily for the first week and twice weekly thereafter following the guidelines of the European Respiratory Society for as long as the infant remained mechanically ventilated (19). The lungs were lavaged with two aliquots of $0.9 \%$ saline solution, $1 \mathrm{~mL} / \mathrm{kg}$ bodyweight and the fluid pooled in the same suction trap.

The BAL fluid was centrifuged immediately at $8000 \mathrm{rpm}$ for $5 \mathrm{~min}$. Supernatant was transferred into small tubes (Eppendorf, Hamburg; Germany) and frozen immediately at $-70^{\circ} \mathrm{C}$.

Cytokine analysis. Cytokine concentration in the supernatant of $78 \mathrm{BAL}$ samples was measured in duplicate using the novel Bio-Plex Protein Array system (BioRad, Hemel Hempstead, Hertfordshire, UK). Multiple bead assay cytokine analysis is based on the simultaneous recognition of fluorescent polystyrene microsheres and quantification of cytokine concentration by dual laser flow cytometry. BAL fluid supernatant was mixed with carboxylated $5.5-\mu \mathrm{m}$ polystyrene beads coated with capture antibody specific against IL-4, IL- 8 , IFN- $\gamma$, TNF- $\alpha$, macrophage chemoattractant protein (MCP)-1, MIP-1 $\beta$ and the anti-inflammatory cytokine IL-10. Following incubation with biotinylated detection antibody and streptavidin R-phycoerythrin dye data were acquired with the Bio-Plex flow cytometer. Data analysis was performed using Bio-Plex Manager 3.0 software using five parametric curve fitting. All procedures were performed following the manufacturer's instructions.

Data collection. The following demographic data were collected: Gestation, birth weight, Apgar scores (at one and five minutes) mode of delivery, length of requirement for mechanical ventilation, and the need for supplemental oxygen until discharge from hospital. Infants transferred back to their local hospital were followed-up with weekly telephone calls until discharge.

Statistical analysis. Based on previous data (5) a difference in stable maximal baseline PD of five $\mathrm{mV}$ between the two cohorts (CLD and non-CLD) was considered clinically significant (estimated SD, $6 \mathrm{mV}$ ). Forty infants were needed to achieve $80 \%$ power at a significance level of 0.05 . Data are presented as medians and ranges or interquartile ranges (IQR). We carefully examined histograms and skewness and also performed a ShapiroWilk tests on all the nasal PD data obtained from the CLD and non-CLD group. Some of the data obtained for stable maximal baseline PDs, change in $\mathrm{PD}$ following amiloride and following zeroCl ${ }^{-}$solutions was not normally distributed (Skewness $>1$ and Shapiro-Wilk $p<0.05$ ) especially those relating to the CLD group. We therefore used nonparametric tests throughout. The Wilcoxon signed-rank test was used to analyze paired data. Comparisons of measurements within subjects were made using the Mann Whitney U-test. Partial regression was used to examine the influence of gestation. Values for $p<0.05$ were deemed statistically significant. Continuous data were compared using Spearman's rank correlation. SPSS for windows version 10 (Chicago, USA) was used for the analysis.

\section{RESULTS}

Subjects. Forty infants born between 23 and 29 wk gestation were recruited into the study (male, $n=24$ ). Characteristics of the study population are shown in Table 1.

Four infants died of respiratory failure before 36 wk corrected gestation (on postnatal day 2, 3, 8 and 41). These infants had severe respiratory distress and none had been
Table 1. Characteristics of the study groups

\begin{tabular}{lcc}
\hline & $\begin{array}{c}\text { CLD } \\
(n=30)\end{array}$ & $\begin{array}{c}\text { No CLD } \\
(n=10)\end{array}$ \\
\hline Male (n) & 19 & 5 \\
Gestation (weeks) & $27(25 ; 28)$ & $28(26 ; 29)$ \\
Birth weight $(\mathrm{g})$ & $860(716 ; 1022)$ & $1138(850 ; 1323)^{*}$ \\
Apgar score $(1 \mathrm{~min})$ & $5(4 ; 7)$ & $5(4 ; 9)$ \\
Apgar score (5 min) & $9(7 ; 9)$ & $8(7 ; 9)$ \\
Time to first successful & $3(2 ; 6)$ & $2(1 ; 4)$ \\
$\quad$ extubation ( $>24$ h) (days) & & 6 \\
Vaginal delivery (n) & 12 & 2 \\
Caesarean section (labor) (n) & 3 &
\end{tabular}

* Significantly different from the CLD group (Mann-Whitney).

CLD, babies who developed chronic lung disease or died before 36 weeks corrected gestation.

All data presented as medians (IQR).

breathing spontaneously off the ventilator before death. Of the 36 infants who survived to $36 \mathrm{wk}$ corrected gestation, 26 (72\%) developed CLD.

The study group comprised five sets of twins born at 26, 27 , 29 and two sets born at 28 wk gestation. Six infants of three pairs of twins developed CLD, the four infants of the other sets did not.

Three babies had intrauterine growth retardation (birth weight $<3^{\text {rd }}$ centile corrected for prematurity). Four infants had significant PDAs, three of which required surgical closure and one was treated with repeated courses of indomethacin (all four developed CLD). One baby was treated for pneumothorax and required a chest drain. In five infants there was evidence clinically for chorioamnionitis with maternal temperature, raised CRP and white blood cell counts. One child had experienced ruptured fetal membranes since $11 \mathrm{wk}$ gestation and required repeated amniotic fluid infusions. Born at 27 wk postmenstrual age the baby had very severe lung disease and never successfully weaned off the ventilator. A degree of pulmonary hypoplasia may have been present. Two extremely preterm infants born at 23 and $25 \mathrm{wk}$ gestation respectively suffered pulmonary hemorrhages and both subsequently died. Six infants were treated postnatally for sepsis, a further infant had proven fungal sepsis. These complications are known to increase the risk for the development of CLD (20).

Thirty-seven infants (23 male) had matched nasal PD measurements and BAL cytokine concentrations. Seventy-eight matched pairs of nasal PD and BAL fluid were available for analysis.

Nasal PD measurement. A total of 129 nasal PD studies were performed on median postnatal day 1, 3, 8 and 29. (Table 2) These will be referred to as day $1,3,8$ and 29 measurements, respectively. The median time to complete the nasal PD measurement from the point of nasal PD recording was 12 min (IQR 10-14).

Two infants on nasal prong continuous positive airway pressure ventilation at the time of nasal PD measurement were switched to facemask oxygen for the duration of the nasal PD study.

Drug treatment. The mothers of all but one infant received antenatal steroids. The mothers of 27 infants were given two doses of betamethasone $12 \mathrm{~h}$ apart, one mother had two 
Table 2. Stable maximal baseline PD, $\delta$-amil ( $\mathrm{mV}$ and \%) and $\delta$-zeroCl - on the first postnatal day, on median postnatal day 3 , 8 and 29 for the whole cohort

\begin{tabular}{lcccc}
\hline & PN day 1 & PN day 3 & PN day 8 & PN day 29 \\
\hline Stable max PD $(\mathrm{mV})$ & $-17.5(+2 ;-53) n=40$ & $-13.0(-2 ;-36) * n=37$ & $-17.0(-7 ;-43) n=33$ & $-19.0(-9 ;-30) n=19$ \\
$\delta$-amil $(\mathrm{mV})$ & $15.5(3 ; 46) n=38$ & $11.0(3 ; 28) n=37$ & $10.0(2 ; 34) n=33$ & $11.0(2 ; 16) n=19$ \\
$\delta$-amil $(\%)$ & $73(33 ; 100) n=38$ & $72(29 ; 100) n=37$ & $61(20 ; 83) * n=33$ & $50(22 ; 84) * n=19$ \\
$\delta$-zeroCl ${ }^{-}(\mathrm{mV})$ & $-1.0(+6 ;-13) n=37$ & $-2.0(+2 ;-15) n=36$ & $-4.0(+5 ;-23) * n=33$ & $-2.0(+3 ;-23) n=19$ \\
\hline
\end{tabular}

PN, median postnatal day; Stable max PD, stable maximal baseline PD; $\delta$-amil (\%): percent inhibition of stable maximal baseline PD following perfusion with amiloride $10^{-4} \mathrm{M} ; \delta$-amil $(\mathrm{mV})$ : change in PD from stable maximal baseline PD following perfusion with amiloride $10^{-4} \mathrm{M} ; \delta$-zeroCl ${ }^{-}(\mathrm{mV})$ : change in PD following perfusion with zeroCl ${ }^{-}$solution and amiloride $10^{-4} \mathrm{M}$.

* Significantly different from postnatal day 1 (Wilcoxon Signed Ranks Test; $p<0.05$ ).

All values given as medians (range).

courses and the remainder $(n=12)$ received only one dose. Median time at birth since the last maternal steroid dose was $60 \mathrm{~h}$ (range 2-189) in the non-CLD group and $55 \mathrm{~h}$ (range 40 min-344 h) in the CLD group. The difference between the groups was not significant (Mann-Whitney).

All the infants requiring intubation and mechanical ventilation received postnatal exogenous surfactant and intravenous antibiotics. Babies under $1000 \mathrm{~g}$ birth weight also received a three-day course of indomethacin to promote closure of the ductus arteriosus. Before extubation babies received a loading dose of caffeine followed by a regular daily dose until they reached $34 \mathrm{wk}$ corrected gestation and no further apnoeas were observed.

Four babies received inotrope (adrenaline) infusions. Three babies were born at 23 and one at 25 wk gestation. Only one of those babies survived beyond the first postnatal week.

Only one child received postnatal oral dexamethasone during the study period. This was started four days before the final (day 29) nasal PD study in this infant. The same child also received oral diuretics (hydrochlorthiazide and spironolactone) started two days before the day 29 nasal PD study.

Severity of the RDS. Babies who required intubation and mechanical ventilation for more than $48 \mathrm{~h}$ following birth had significantly lower stable maximal baseline PD (MannWhitney; $p<0.05$ ). Amiloride sensitive PD (in $\mathrm{mV}$ ) was also lower $(p<0.05)$. These differences were no longer significant when corrected for gestational age $(p=0.138$ and $p=0.149$, respectively).

Relationship between ion transport parameters and gestation. Stable maximal baseline PD on the first postnatal day significantly increased with gestational age. (Fig. 1) Most of this PD was amiloride sensitive (median inhibition 73\%) suggesting predominantly $\mathrm{ENaC}$ mediated $\mathrm{Na}^{+}$absorption. (Table 2) On postnatal day three the stable maximal baseline PD was significantly lower (Fig. 2) and no longer associated with gestation.The majority of this PD however remained amiloride sensitive. Babies of all gestational age groups moved toward a less amiloride sensitive PD over the first four postnatal weeks. On postnatal day 29 both, the change in $\mathrm{mV}$ and the percentage change from stable maximal baseline PD were significantly different from the postnatal day 1 values (Table 2).

The capacity of the postnatal airway epithelium to secrete $\mathrm{Cl}^{-}$was very low at all gestational ages and did not change much over the study period.

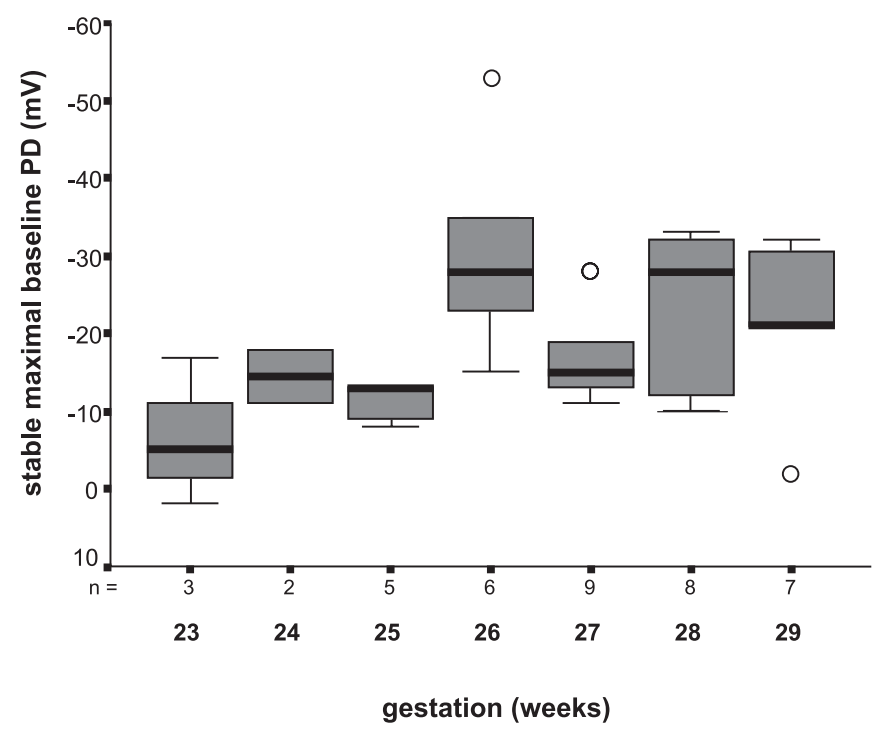

Figure 1. Stable maximal baseline PD on the first postnatal day $(n=40)$. The boxes represent the median (solid line) and the IQR. Whiskers represent the range and circles are outliers. Stable maximal baseline PD significantly correlated with gestational age (Spearman's rank correlation coefficient; $p<$ $0.05)$

Relationship between ion transport parameters and CLD. Stable maximal baseline PDs following birth were similar in CLD and non-CLD infants.(Fig. 3) There was no difference also in the amiloride sensitivity of this PD, which was very high. Whereas the stable maximal baseline PD in CLD infants remained virtually unchanged throughout the study period, non-CLD infants had a significantly greater stable maximal baseline PD on postnatal day 29. (Fig. 3) By this time the amiloride sensitive portion of stable maximal baseline PD was lower in both cohorts compared to postnatal day 1 .

Changes in PD following perfusion with $\mathrm{zeroCl}^{-}$were small throughout the first four postnatal weeks in CLD and non-CLD infants. Non-CLD babies had a significantly greater capacity for $\mathrm{Cl}^{-}$secretion on postnatal day one, but the absolute difference was small. (Table 3) The CLD group demonstrated a significantly increased capacity for $\mathrm{Cl}^{-}$secretion on postnatal day three and eight. The absolute changes were again small.

Cytokine concentration in BAL fluid. With the exception of two samples all BAL fluid was collected within $24 \mathrm{~h}$ of the nasal PD measurement. Concentrations of all cytokines rose by postnatal day eight but the difference was only significant 


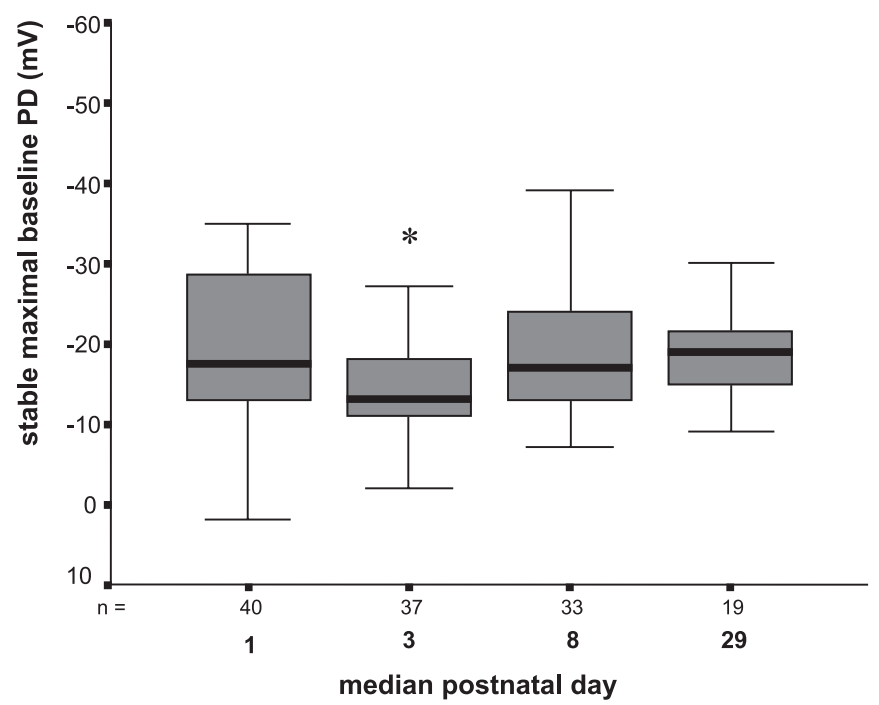

Figure 2. Median max baseline PD for all extremely preterm infants born at 23 to 29 wk gestation on postnatal day 1 , day 3 , day 8 and day 29 . * Significantly different from baseline (Wilcoxon Signed Ranks Test $p<0.05$ )

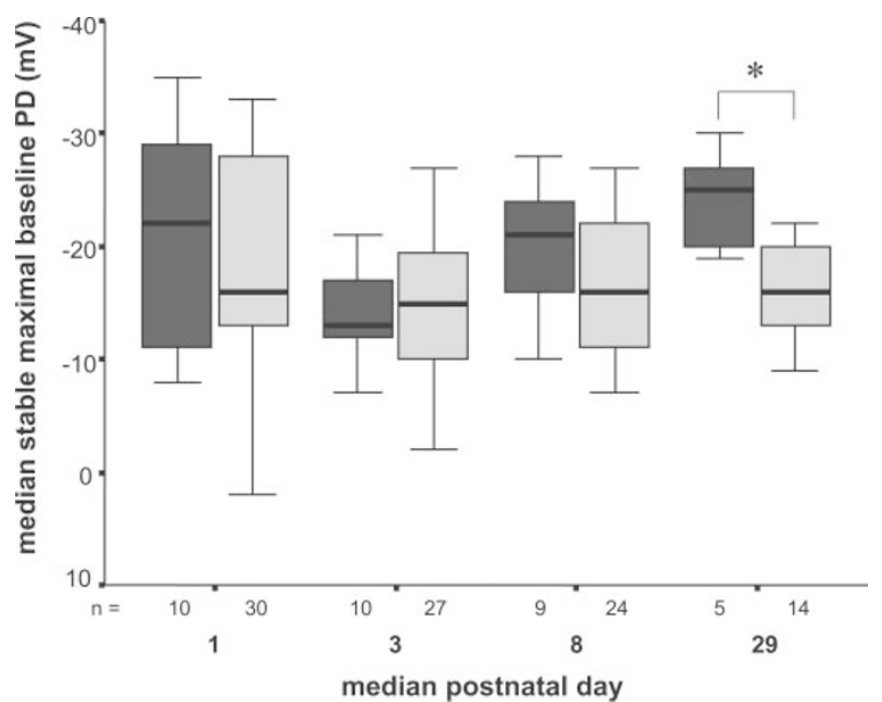

Figure 3. Median stable maximal baseline PD on the first postnatal day, on postnatal day 3, 8 and 29 for CLD and non-CLD infants. Dark grey boxes represent non-CLD and light grey boxes CLD infants. Non-CLD infants had a significantly greater (Mann-Whitney; $p<0.05$ ) stable maximal baseline PD on postnatal day 29 only (*)

for IL-8 and IFN- $\gamma$. Cytokine concentrations in BAL fluid were no different between CLD and non-CLD infants on the first and third postnatal day (data not shown). BAL fluid of only three non-CLD infants was available on postnatal day three and of only one infant on postnatal day eight (Table 4).

Cytokine concentration and airway ion transport. Detailed comparison was made between cytokine concentrations in BAL fluid and proximal airway ion transport parameters obtained from the nasal PD measurement. We studied the scatterplots and regression lines of airway ion transport parameters (stable maximal baseline $\mathrm{PD}, \delta$-amiloride expressed as a percentage and in absolute values (mV) and $\delta$-zeroCl ${ }^{-}$) and each cytokine concentration on postnatal day one, three, eight and 29. A total of 112 scatterplots were studied. Spear- man's rank correlation was used to examine the association between airway ion transport parameters and cytokine concentrations.

When comparing cytokine concentrations in BAL fluid and proximal airway ion transport parameters obtained from the nasal PD measurement, there was no apparent association between the concentration of any cytokine in BAL (IL-4, IL-8, TNF- $\alpha$, IFN- $\gamma$, IL-10, MCP-1 and MIP-1 $\beta$ ) and airway ion transport parameters (stable maximal baseline PD $(\mathrm{mV}), \delta$-amil $(\mathrm{mV})$, $\delta$-amil $(\%)$ and $\delta$-zeroCl $\left.{ }^{-}(\mathrm{mV})\right)$ at any time point.

\section{DISCUSSION}

Active $\mathrm{Cl}^{-}$secretion by the fetal airway epithelium creates the osmotic gradient for the passive flow of liquid into the lung lumen resulting in small increases in distension pressure essential for normal fetal lung growth. At the time of term birth $\mathrm{Cl}^{-}$secretion into the lung lumen slows. Apical membrane $\mathrm{Na}^{+}$absorption, facilitated by the amiloride sensitive epithelial $\mathrm{Na}^{+}$channel $(\mathrm{ENaC})$ becomes the predominant airway ion transport process across the respiratory epithelium leading to lung liquid reabsorption from the bronchial and alveolar lumen essential for postnatal adaptation to air breathing $(3,4,21-23)$.

Data on postnatal airway ion transport in preterm human babies are very limited. To our knowledge no data of postnatal airway ion transport in infants who develop CLD or in relation to airway inflammation have been published.

Nasal potential difference measurements have been used in neonates $(5,17,24)$ and in adults with cystic fibrosis $(25)$ as a proxy measure to examine airway ion transport throughout the respiratory epithelium. The epithelium along the floor of the nose of neonates is formed by cuboidal, ciliated cells, which are very similar morphologically and in their ion transport characteristics to those found in the lower respiratory tract (26). The PD measured reflects the sum of ion transport processes across the epithelium. Ohm's law states that there is a linear relation between electrical flow and its driving force (the potential difference).

We found that stable maximal baseline PD across the nasal epithelium increases with gestational age and that this PD postnatally is largely amiloride sensitive at all gestational ages. This suggests that there has been a very effective switch to a postnatal absorptive pattern of airway ion transport even in the most immature babies. This also strongly indicates that although very preterm infants have less absorptive airway ion transport compared with more mature babies, the majority of it is $\mathrm{ENaC}$ mediated.

We have previously published nasal PD data obtained on postnatal day one in moderately preterm infants (27). Compared with babies born $>30$ wk gestation stable maximal baseline PD was lower in infants $<30$ wk gestation. This steady increase in nasal PD peaked at around 34 to $36 \mathrm{wk}$ gestation. We speculate that in preterm infants there is maximal activation of absorptive airway ion transport, the magnitude of which increases in a gestation dependent manner due to increased expression of $\mathrm{ENaC} \mathrm{(28).}$ 
Table 3. Stable maximal baseline PD, $\delta$-amil $(\mathrm{mV}$ and \%) and $\delta$-zeroCl - on postnatal days 1, 3, 8 and 29 in CLD and non-CLD infants

\begin{tabular}{|c|c|c|c|c|c|c|c|c|}
\hline & \multicolumn{2}{|c|}{ PN day 1} & \multicolumn{2}{|c|}{ PN day 3} & \multicolumn{2}{|c|}{ PN day 8} & \multicolumn{2}{|c|}{ PN day 29} \\
\hline & CLD & No CLD & CLD & No CLD & CLD & No CLD & CLD & No CLD \\
\hline $\begin{array}{l}\text { Stable maximal } \\
\text { baseline PD } \\
(\mathrm{mV})\end{array}$ & $-16(+2 ;-53)$ & $-22(-8 ;-35)$ & $-15(-2 ;-36)$ & $-13(-7 ;-21)$ & $-16(-7 ;-43)$ & $21(-10 ;-28)$ & $-16(-9 ;-22)$ & $-25(-19 ;-30)^{*}$ \\
\hline$n$ & 30 & 10 & 27 & 10 & 24 & 9 & 14 & 5 \\
\hline$\delta$-amil (mV) & $15(3 ; 46)$ & $16.5(7 ; 29)$ & $11(3 ; 28)$ & $10(5 ; 17)$ & $10(2 ; 34)$ & $12(5 ; 20)$ & $10.5(2 ; 15)$ & $13(10 ; 16)$ \\
\hline$n$ & 28 & 10 & 27 & 10 & 24 & 9 & 14 & 5 \\
\hline$\delta$-amil (\%) & $-73(-33 ;-100)$ & $-73(-52 ;-100)$ & $-73(-46 ;-100)$ & $-71(-29 ;-100)$ & $-67(-20 ;-83)$ & $54(-38 ;-83)$ & $-61(-22 ;-75)$ & $-48(-44 ;-84)$ \\
\hline$n$ & 28 & 10 & 27 & 10 & 24 & 9 & 14 & 5 \\
\hline $\begin{array}{c}\delta \text {-zeroCl- } \\
(\mathrm{mV})\end{array}$ & $-1.0(+6 ;-10)$ & $-2.0(+2 ;-13)^{*}$ & $-2.5(+2 ;-15) \dagger$ & $-2.0(+1 ;-10)$ & $-5.0(+5 ;-23) \dagger$ & $-2.0(0 ;-6)$ & $-2.0(+3 ;-23)$ & $1.0(3 ;-6)$. \\
\hline$n$ & 28 & 9 & 26 & 10 & 24 & 9 & 14 & 5 \\
\hline
\end{tabular}

PN, median postnatal day.

* Significantly different from CLD; † significantly different from postnatal day one.

All data given as median (range).

Table 4. Cytokine concentration on median postnatal day 1, 3, 8 and 29 in infants born before 30 wpma

\begin{tabular}{lcccr}
\hline & PN day 1 & PN day 3 & PN day 8 & PN day 29 \\
\hline IL-4 $(n)$ Range & $256(35)(38-1326)$ & $206(19)(80-1533)$ & $351(14)(73-1660)$ & $190(7)(89-517)$ \\
IL-8 $(n)$ Range & $3453(34)(1514-6873)$ & $3907(18) \dagger(1358-7813)$ & $3989(13) \dagger(2595-7349)$ & $3466(8)(2632-7169)$ \\
TNF- $\alpha(n)$ Range & $547(37)(110-509)$ & $529(19)(207-2403)$ & $1335(14)(232-7676)$ & $762(8)(266-3787)$ \\
IFN- $\gamma(n)$ Range & $648(36)(211-1988)$ & $543(19)(296-2085)$ & $774(14) \dagger(269-2562)$ & $456(7)(312-980)$ \\
IL-10 $(n)$ Range & $<$ OOR $(35)(<$ OOR-633) & $<$ OOR $(19)(<$ OOR-676) & $12(14)(<$ OOR-2699) & $<$ OOR $(8)(<$ OOR-115) \\
MCP-1 $(n)$ Range & $3825(32)(1124-8263)$ & $4912(18)(862-8786)$ & $4932(12)(1769-9643)$ & $4861(7)(536-7307)$ \\
MIP-1 $\beta(n)$ Range & $4233(34)(1261-8412)$ & $3699(17)(699-8566)$ & $4567(11)(1690-8235)$ & $3995(7)(1454-5948)$ \\
\hline
\end{tabular}

Values given as medians (range). Cytokine concentrations in $\mathrm{pg} / \mathrm{mL}$.

$\dagger$ Significantly different from postnatal day 1 .

PN, median postnatal day; wpma, weeks postmenstrual age; IL, interleukin; TNF, tumor necrosis factor; IFN, interferon.

Our findings are also consistent with a previous study involving very preterm infants (5). Compared with the study reported by Barker et al. we found a greater median proportion of $\mathrm{ENaC}$ mediated PD on the first postnatal day following perfusion with amiloride (39\% versus $73 \%$ ). This is likely to be the result of the higher concentration of amiloride used in our study $\left(10^{-4} \mathrm{M}\right.$ compared with $\left.10^{-5} \mathrm{M}\right)$. A number of studies support the use of amiloride $10^{-4} \mathrm{M}$ to achieve complete $\mathrm{ENaC}$ inhibition $(17,25)$.

On postnatal day one and three, median inhibition of PD following amiloride $\left(10^{-4} \mathrm{M}\right)$ was high for the cohort as a whole (median 73\%). This fell gradually to a median of $50 \%$ on postnatal day 29 , which was significantly lower compared with postnatal day one, and may reflect an increase in amiloride insensitive $\mathrm{Na}^{+}$transport. Similar findings were reported in newborn guinea pigs, which have the greatest amiloride sensitive lung liquid clearance immediately after birth followed by a fall to adult levels within five days (29). Transport of other ions however cannot be entirely ruled out. The PD across the airway epithelium reflects the sum of all the ion transport processes taking place. With the use of amiloride we can reliably only make assumptions about the apical $\mathrm{Na}^{+}$transport.

Stable maximal baseline PD was not significantly different between CLD and non-CLD babies in the early postnatal period. However, on postnatal day 29 non-CLD babies had a significantly higher stable maximal baseline PD. This PD remained very low throughout the study period in CLD infants. These longitudinal data raise the possibility that preterm infants who develop CLD may have a persistently reduced or inhibited postnatal absorptive airway ion transport capacity compared with non-CLD babies. The amiloride sensitive portion of the stable maximal baseline PD at each time point was very similar in both cohorts over the first four postnatal weeks. The numbers of non-CLD babies with nasal PD studies was small after four postnatal weeks as we had to rely on intravenous access devices to perform nasal PD studies.

In our cohort of preterm infants there was little capacity for $\mathrm{Cl}^{-}$secretion across the proximal airway epithelium throughout the study period. On postnatal day eight infants who subsequently developed CLD had significantly greater $\mathrm{Cl}^{-}$secretory capacity but this nevertheless remained very low compared with normal adult levels (25). We were unable to examine the impact of cAMP agonists such as isoprenaline on $\mathrm{Cl}^{-}$secretion because of concerns about a systemic effect from these drugs.

Preterm infants with RDS in our study developed airway inflammation. We measured lung lavage fluid concentration of cytokines employing a multiple bead assay cytokine analysis, which has a good correlation with standard ELISA (30). Concentration of all seven cytokines in BAL rose following birth, although this was only significant for IFN- $\gamma$ and IL-8, reaching a peak level on postnatal day eight consistent with previous studies of preterm infants $(8-11)$. We found no difference in the concentration of any cytokine between CLD and non-CLD babies. This difference however is most marked at the end of the first postnatal week at which point we were only able to obtain BAL fluid from three non-CLD babies.

We did not demonstrate an association between airway ion transport processes and lung inflammatory markers in our cohort 
of very preterm babies. Ideally the two measurements would have come from the same compartment but it is currently not possible to measure airway ion transport in the distal airway of newborn infants in vivo. There is some evidence that cytokines increased in lung fluid are also increased in peripheral blood $(31,32)$ but this was not measured in this study.

It is possible that, in an effort to clear lung liquid following birth, the absorptive airway ion transport capacity of the newborn preterm airway is maximally activated by factors like for example high circulating catecholamine levels. Further modulation by pro-inflammatory cytokines may not take place at this early stage. However, we did find increasing absorptive airway ion transport capacity in preterm infants who did not develop CLD. The timing coincides with the decrease in airway inflammation reported for preterm non-CLD infants after the first postnatal week and this merits further study.

Study limitations: A larger than expected number of infants developed CLD compared with previous unit records. This was not associated with any change in unit policy. The characterisation of the chloride secretory capacity of the airway was limited by not being able to include isoprenaline in the perfusate. Infants without venous access were not measured. This was most pertinent at the end of the study period leading to incomplete data sets. Finally twin pairs were included with the potential confounding affect on data analysis (although initial subgroup analysis did not support this).

\section{CONCLUSION}

Despite the challenge of examining airway ion transport processes in preterm infants we have generated data that give more insight into the adaptive lung liquid absorptive capabilities of these babies at birth.

We found that: 1) although preterm infants have a reduced stable maximal baseline PD, they demonstrate clear evidence of a switch from a secretory to an absorptive airway phenotype; 2) infants with CLD had persistently lower postnatal nasal PDs; and 3) no relationship between airway inflammation and reduced absorptive airway ion transport in infants with CLD.

This study supports previous work suggesting a reduced lung liquid absorptive capacity in extremely preterm babies, although even in the most premature babies the switch to a postnatal airway ion transport phenotype is evident. The relationship between ion transport and inflammation needs to be examined in an animal model of preterm birth.

\section{REFERENCES}

1. Gaillard EA, Shaw BN 2003 Chronic lung disease in infancy following prematurity. Hosp Med 64:640-643

2. Lorenz JM 2001 The outcome of extreme prematurity. Semin Perinatol 25:348-359

3. O'Brodovich H, Hannam V, Seear M, Mullen JB 1990 Amiloride impairs lung water clearance in newborn guinea pigs. J Appl Physiol 68:1758-1762

4. Olver RE, Walters DV, Wilson M 2004 Developmental regulation of lung liquid transport. Annu Rev Physiol 66:77-101

5. Barker PM, Gowen CW, Lawson EE, Knowles MR 1997 Decreased sodium ion absorption across nasal epithelium of very premature infants with respiratory distress syndrome. J Pediatr 130:373-377
6. DeSa DJ 1969 Pulmonary fluid content in infants with respiratory distress. J Pathol 97:469-478

7. Adams EW, Counsell SJ, Hajnal JV, Cox PN, Kennea NL, Thornton AS, Bryan AC, Edwards AD 2002 Magnetic resonance imaging of lung water content and distribution in term and preterm infants. Am J Respir Crit Care Med 166:397-402

8. Beresford MW, Shaw NJ 2002 Detectable IL-8 and IL-10 in bronchoalveolar lavage fluid from preterm infants ventilated for respiratory distress syndrome. Pediatr Res 52:973-978

9. Bagchi A, Viscardi RM, Taciak V, Ensor JE, McCrea KA, Hasday JD 1994 Increased activity of interleukin- 6 but not tumor necrosis factor-alpha in lung lavage of premature infants is associated with the development of bronchopulmonary dysplasia. Pediatr Res 36:244-252

10. Kotecha S, Wilson L, Wangoo A, Silverman M, Shaw RJ 1996 Increase in interleukin (IL)-1 beta and IL-6 in bronchoalveolar lavage fluid obtained from infants with chronic lung disease of prematurity. Pediatr Res 40:250-256

11. Speer CP 2004 Pre- and postnatal inflammatory mechanisms in chronic lung disease of preterm infants. Paediatr Respir Rev 5:S241-S244

12. Galietta LJ, Pagesy P, Folli C, Caci E, Romio L, Costes B, Nicolis E, Cabrini G, Goossens M, Ravazzolo R, Zegarra-Moran O 2002 IL-4 is a potent modulator of ion transport in the human bronchial epithelium in vitro. J Immunol 168:839-845

13. Galietta LJ, Folli C, Marchetti C, Romano L, Carpani D, Conese M, Zegarra-Moran O 2000 Modification of transepithelial ion transport in human cultured bronchial epithelial cells by interferon-gamma. Am J Physiol Lung Cell Mol Physiol 278:L1186-L1194

14. Borjesson A, Norlin A, Wang X, Andersson R, Folkesson HG 2000 TNF-alpha stimulates alveolar liquid clearance during intestinal ischemia-reperfusion in rats. Am J Physiol Lung Cell Mol Physiol 278:L3-L12

15. Nair PK, Li T, Bhattacharjee R, Ye X, Folkesson HG 2005 Oxytocin-induced labor augments IL-1-stimulated distal lung fluid absorption in fetal guinea pig lungs. Am J Physiol Lung Cell Mol Physiol 289:L1029-L1038

16. Folkesson HG, Pittet JF, Nitenberg G, Matthay MA 1996 Transforming growth factor-alpha increases alveolar liquid clearance in anesthetized ventilated rats. Am J Physiol 271:L236-L244

17. Southern KW, Noone PG, Bosworth DG, Legrys VA, Knowles MR, Barker PM 2001 A modified technique for measurement of nasal transepithelial potential difference in infants. J Pediatr 139:353-358

18. Gaillard EA, Shaw NJ, Subhedar NV, Wallace HL, Southern KW 2003 Airway ion transport on the first postnatal day in infants delivered vaginally or by elective cesarean section. Pediatr Res 54:58-63

19. de Blic J, Midulla F, Barbato A, Clement A, Dab I, Eber E, Green C, Grigg J, Kotecha S, Kurland G, Pohunek P, Ratjen F, Rossi G 2000 Bronchoalveolar lavage in children. ERS Task Force on bronchoalveolar lavage in children. European Respiratory Society. Eur Respir J 15:217-231

20. Rojas MA, Gonzalez A, Bancalari E, Claure N, Poole C, Silva-Neto G 1995 Changing trends in the epidemiology and pathogenesis of neonatal chronic lung disease. J Pediatr 126:605-610

21. O'Brodovich H 2004 Deficient $\mathrm{Na}+$ channel expression in newborn respiratory distress syndrome. Pediatr Pulmonol Suppl 26:141-142

22. Hummler E, Barker P, Gatzy J, Beermann F, Verdumo C, Schmidt A, Boucher R, Rossier BC 1996 Early death due to defective neonatal lung liquid clearance in alpha-ENaC-deficient mice. Nat Genet 12:325-328

23. Helve O, Pitkanen OM, Andersson S, O'Brodovich H, Kirjavainen T, Otulakowski G 2004 Low expression of human epithelial sodium channel in airway epithelium of preterm infants with respiratory distress. Pediatrics 113:1267-1272

24. Gowen CW, Lawson EE, Gingras J, Boucher RC, Gatzy JT, Knowles MR 1988 Electrical potential difference and ion transport across nasal epithelium of term neonates: correlation with mode of delivery, transient tachypnea of the newborn, and respiratory rate. J Pediatr 113:121-127

25. Knowles MR, Olivier KN, Hohneker KW, Robinson J, Bennett WD, Boucher RC 1995 Pharmacologic treatment of abnormal ion transport in the airway epithelium in cystic fibrosis. Chest 107:S71-S76.

26. Knowles M, Murray G, Shallal J, Askin F, Ranga V, Gatzy J, Boucher R 1984 Bioelectric properties and ion flow across excised human bronchi. J Appl Physiol 56:868-877

27. Gaillard EA, Shaw NJ, Wallace HL, Subhedar NV, Southern KW 2005 Nasal potential difference increases with gestation in moderately preterm neonates on the first postnatal day. Arch Dis Child Fetal Neonatal Ed 90:F172-F173

28. Smith DE, Otulakowski G, Yeger H, Post M, Cutz E, O’Brodovich HM 2000 Epithelial $\mathrm{Na}(+)$ channel $(\mathrm{ENaC})$ expression in the developing normal and abnormal human perinatal lung. Am J Respir Crit Care Med 161:1322-1331

29. Finley N, Norlin A, Baines DL, Folkesson HG 1998 Alveolar epithelial fluid clearance is mediated by endogenous catecholamines at birth in guinea pigs. J Clin Invest 101:972-981

30. de Jager W, te Velthuis H, Prakken BJ, Kuis W, Rijkers GT 2003 Simultaneous detection of 15 human cytokines in a single sample of stimulated peripheral blood mononuclear cells. Clin Diagn Lab Immunol 10:133-139

31. Wiedermann FJ, Mayr AJ, Kaneider NC, Fuchs D, Mutz NJ, Schobersberger W 2004 Alveolar granulocyte colony-stimulating factor and alpha-chemokines in relation to serum levels, pulmonary neutrophilia, and severity of lung injury in ARDS. Chest 125:212-219

32. Mak JC, Ho SP, Leung RY, Ho PL, Ooi C, Tipoe GL, Yan C, Ip MS, Lam WK, Tsang KW 2005 Elevated levels of transforming growth factor-beta(1) in serum of patients with stable bronchiectasis. Respir Med 99:1223-1228 